\title{
BER Performance Analysis of Cognitive Radio Network Using M-ary PSK over Rician Fading Channel.
}

\author{
Ammar Izzeldin Mohammed ${ }^{\mathbf{1}}$, Khalid Hamid Bilal ${ }^{\mathbf{2}}$, Mohammed Faisal Osman ${ }^{3}$ \\ ${ }^{1,2}$ University of Sciences \& Technology Faculty of Engineering Khartoum-Sudan \\ ${ }^{3}$ Karary University Khartoum-Sudan ${ }^{3}$
}

\begin{abstract}
This paper, focus on the performance evaluation of cognitive radio network in terms of BER VS SNR for M-ary PSK modulation schemes, such as BPSK, QPSK, 8-PSK, 16-PSK, 32-PSK, and 64-PSK over AWGN and Rician fading channel. The cognitive radio network was designed firstly as a computer model showing the sequence of processes in cognitive radio system and then the Simulation of cognitive radio work was performed using MATLAB simulation. The results were obtained in terms of BER VS SNR for M-PSK modulation. The results show that as the number of M-ary increase the BER increased while the BER decreased as the SNR increased, although as Spectrum efficiency increase.
\end{abstract}

Keywords: PU, SU, Holes, Cognitive Radio, AWGN, Rician, BER, SNR, BPSK, QPSK, 8-PSK, 16-PSK, 32-PSKand64PSK.

\section{Introduction}

First one to be attention to the radio waves was James Maxwell. This was done through a mathematical experiment in 1865 . He observed wave properties similar to light and close to electrical and magnetic properties. He proposed equations in which light waves and radio waves were described as electromagnetic waves swimming in the surrounding space. In 1887, the German scientist Hertz showed the validity of Maxwell's electromagnetic waves by experimenting the production of radio waves in the laboratory. Then came many inventions showing how practical it is to use radio waves to transmit information through space. Nicola Tesla and Guillermo Marconi continued their series of research in this field, leading to the discovery of systems that allow use of radio waves in communications. [1] Since then, the telecommunications industry has developed faster than in the past because new and sophisticated products enter the markets day after day. As a result the ability to communicate anywhere, anytime with any information and by any person is became driving force of the electronic communications sector around the world. Therefore it is necessary to establish controls and conditions to regulate the management of spectrum access and management ideally since the process of spectrum management is a set of technical and administrative measures to ensure the orderly and effective performance of various wireless services, without harmful interference. Because RF is not limited by the geographical boundaries of countries. Therefore, the frequency spectrum management activities are characterized by being universal which well be in accordance with international radio systems issued by the International Telecommunication Union (ITU) (it is one of specialized United Nations agencies and specializes in various communications issues at the global level). The allocation of frequencies suitable for all radio services is the basic function of spectrum management in accordance with the laws and standards established by the ITU. This includes various activities: study and analysis frequency assignment requests, wireless licensing and spectrum coordination at the local and international levels. Wireless monitoring is the task of ensuring the orderly and efficient performance of wireless uses and Protection the frequency allocations at a local and international levels from interference with other wireless systems and to ensure that certain allocations do not cause interference to other users. However, due to limited spectrum, there is a problem of the scarcity of available frequency spectrum to meet the requirements of new subscribers. As a result, the research teams are exploring new ways and developing new communication mechanisms that will create new generations of wireless networks to meet all the needs arising from the growth and development of communications recently.

One of the most promising solutions to these problems is a cognitive radio system. J.Motola first one appearance the concept of cognitive radio in his thesis in 1999 [2].Cognitive radio (CR) is a smart radio platform with the ability to exploit its environment to increase spectral efficiency and capacity. Cognitive radio allows the secondary user to use the unused frequency with the Primary user without interference between them in the same frequency band. Secondary user detect the holes in PU band [3] to use it. Spectrum hole defined as a spectral range that can be used by secondary user. There are three type of spectrum holes: Black, Grey and White holes [4] In our study we are going to using the energy detection to detect holes in PU band is used by SU then choose the types of digital modulation to be used by secondary user [5]. Where CR works by processing the data to be transmitted through the radio carrier. There are only three characteristics of a signal that can be changed over time: amplitude, phase, or frequency. By modulating the shape and characteristics, this led to 
greater use of spectrum efficiency and increases the bandwidth. There are many higher order modulation techniques like M-PSK have emerged to achieve this goal. The considerations in selecting a particular process digital modulation technique is [6] is to get the highest data transmission speed, optimized bandwidth usage, minimum power transmit, minimum probability of error bits and Low cost. PSK is the commonly used digital modulation technique. The phase of the carrier In M-PSK modulation is changed to state two or more different signal elements. Both peak amplitude and frequency remain constant as the phase changes [7]. Also the M-ary signaling scheme, represent two or more bits are collected together to form symbols and one of M possible signals, s1 (t), s2 (t)... SM (t) is transmitted during each symbol period of duration Ts. Usually, the number of possible signals is $\mathrm{M}=2^{K}$ where $\mathrm{k}$ is an integer [8]. BPSK is special case of M-PSK modulation, the data rate of send is $1 \mathrm{bit} / \mathrm{Hz}$, so BPSK is spectral efficiency modulation [9] [10]. On the other hand, M-PSK can achieve constant carrier amplitude that it is means more efficient nonlinear power amplification can be used. [11] [12].

This paper presents an analysis of bit error rate (BER) performance over a Rician fading channel with AWGN noise in cognitive radio network by using M-PSK modulation technique's our measurement criteria will depends on Bit Error Rate (BER) VS SNR where BER is a better performance measure to evaluate a Cognitive radio system.

\section{System Model}

The MATLAB Simulink program was used to design a simulation model to stimulate cognitive radio system by measuring the bit error rate (BER) in the data versus to signal to noise ratio (SNR), where BER is the most important factors to determine the quality and efficiency of the system that works on the transfer of digital data. The cognitive radio simulation scheme consists of: PU block, SU block, cognitive radio system, energy detection, error rate calculator with bit rate monitoring error. PU is the principal owner and owner of rights to use spectrum band since it has full powers to use it, other users attempt to use this band if PU is absence figure 1 show the PU steps in simulation.

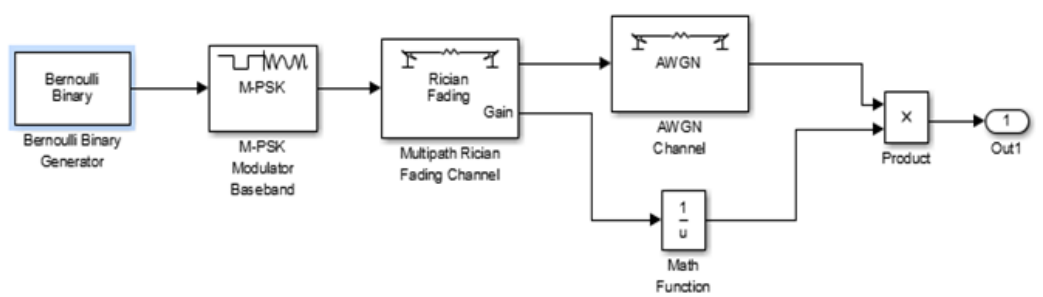

Figure (1): Primary user in Block in Simulink

SU is same technology like PU, the difference only PU has the license. Energy detection one of the important techniques used in Cognitive radio as a guidance of Secondary user to detect white space (hole's) in PU spectrum. The Energy detection was designed to detect radio wave depending on its energy. Figure 2 below shown Energy detection blocks diagram as implemented in the simulation.

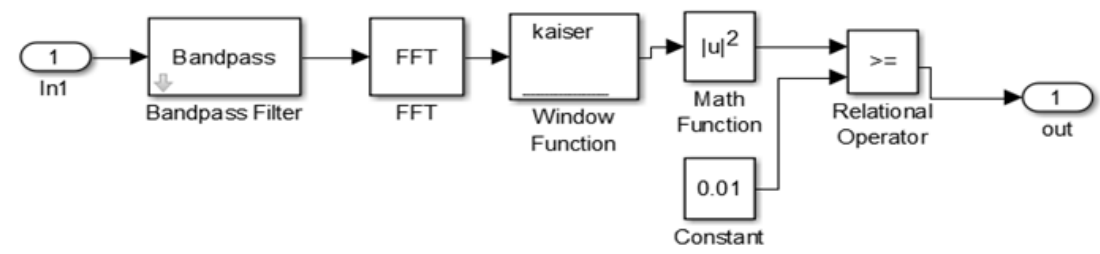

Figure (2): Energy Detector in Simulink

Cognitive radio system adjusts parameters according to the surrounding environment and prevents any interference between the users used same frequency band. The error rate is calculate the sent data of SU by using BER calculation unit in the simulation program.

\section{Mathematical Model.}

The BER performance of any digital modulation scheme generally defined as:

$$
\mathrm{BER}=\frac{\text { Number of Error Bits }}{\text { Number of Total Bits }}
$$

From [13] we determine the probability of error for linear BPSK signaling in Rician fading channels is given as

$$
P_{b}=Q 1(a, b)-\frac{1}{2}\left[1+\sqrt{\frac{d}{d+1}}\right] \exp \left(-\frac{a^{2}+b^{2}}{2}\right) I_{o}(a b)
$$


Where $\mathrm{a}=\left[\frac{K_{r}^{2}[1+2 d-2 \sqrt{d(d+1)}]}{2(d+1)}\right], \mathrm{b}=\left[\frac{K_{r}^{2}[1+2 d+2 \sqrt{d(d+1)}]}{2(d+1)}\right]$

$$
K_{r}=\frac{\alpha^{2}}{2 \sigma^{2}}, \mathrm{~d}=\sigma^{2} \frac{E_{b}}{N_{o}}
$$

\section{Computer Model}

The implementation of cognitive radio system based on status of primary user signal scheme, energy detector unit it's provide status of adjacent environment to the cognitive system and detect the holes in primary frequency, when primary signal detect cognitive radio system will start analysis for a primary user environment and readjust the operator of secondary signal to operate within primary spectrum holes, but if the primary signal is not detected. Energy detector unit will keep searching to determine PU signal scheme. In case of spectrum availability, the secondary user can exploit holes in primary user spectrum; otherwise cognitive radio system set idle. After the cognitive radio system succeeds in using unlicensed spectrum BER is calculated.

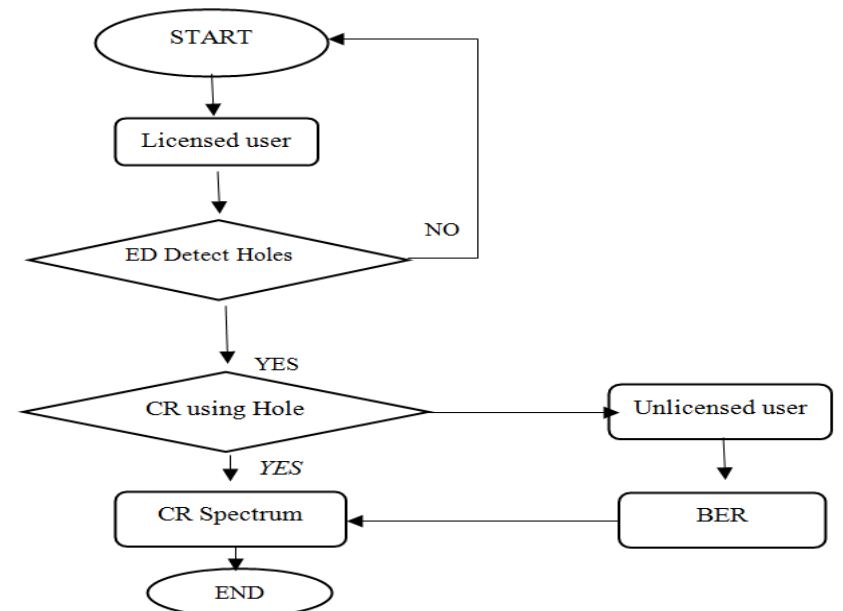

Figure (3): CR Flow Chart Steps.

\section{Simulation Parameter.}

Table I Simulation Parameters

\begin{tabular}{|l|c|}
\hline \multicolumn{1}{|c|}{ Parameter } & Value \\
\hline SNR Range & $0-35 \mathrm{~dB}$ \\
\hline Modulation Technique & M-PSK \\
\hline Channel & Rician fading \\
\hline Sample Time & $1 \mathrm{~s}$ \\
\hline Simulation Time & 24 hours $=86400 \mathrm{~s}$ \\
\hline Data source & Bernoulli binary generator 61 \\
\hline Doppler shift frequency & $0.01 \mathrm{~Hz}$ With Jakes Model \\
\hline Input Signal Power & 1 Watt \\
\hline $\begin{array}{l}\text { Ratio of The Specular Component to the Diffuse } \\
\text { Multipath Components(K factor) }\end{array}$ & 3 \\
\hline
\end{tabular}

\section{Result and Discussion.}

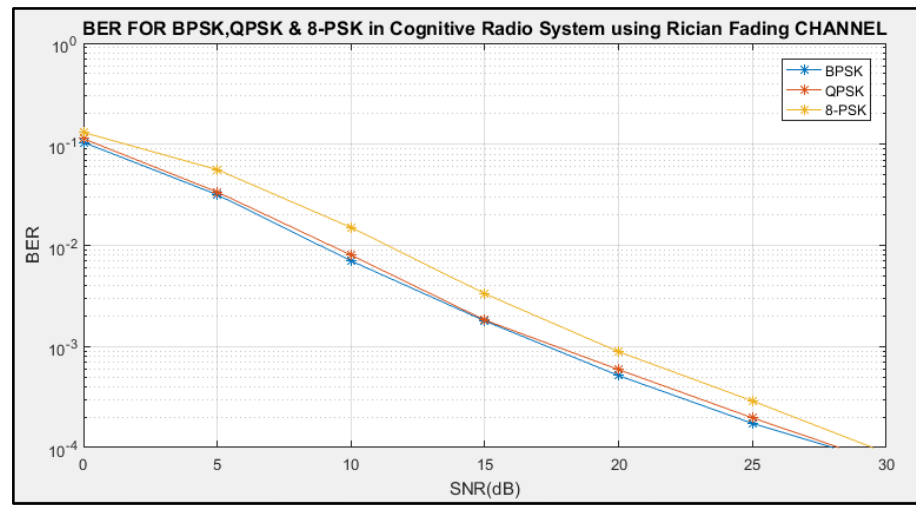

Figure (4): BER FOR BPSK\&QPSK\&8-PSK in Rician fading channel. 
In figure (4) shows BER vs. SNR performance analysis of BPSK, QPSK and 8-PSK modulation technique over Rician fading channels BPSK has lower BER than QPSK and 8-PSK. At SNR of $5 \mathrm{~dB}$, the average BER of BPSK is 0.03172 where QPSK is 0.03377 and 8-PSK is 0.05616 . also at SNR of $30 \mathrm{~dB}$, the average BER of BPSK is equal to the average BER in QPSK it's 0.00006944 where in 8-PSK is equals 0.00008873.The data rate in 8-PSK is greater than BPSK \& QPSK because the symbol rate in 8-PSK is $1 / 3$ and it modulate three bit per symbol that is give highest data rate than BPSK and QPSK.

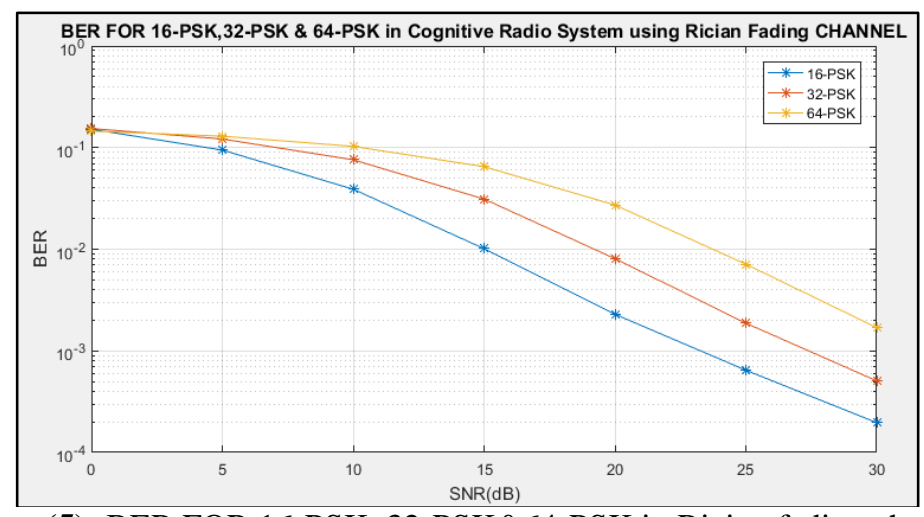

Figure (5): BER FOR 16-PSK, 32-PSK\&64-PSK in Rician fading channel.

Figure (5) shows BER VS SNR performance analysis of 16-PSK, 32-PSK and 64-PSK modulation technique over Rician fading channels and AWGN, 16-PSK has lower BER than 32-PSK and 64-PSK. at SNR of $5 \mathrm{~dB}$ the average BER of 16-PSK is 0.0947 where 32-PSK is equal 0.1217 \& 64-PSK is around 0.1298.also at SNR of $15 \mathrm{~dB}$ the average BER of 16-PSK is equals $10^{-2}(0.01)$ where the average BER of 32-PSK \& 64-PSK is greater than $10^{-2}(0.01)$, the graph shows when SNR value increases, BER is decreases in all three modulation technique.

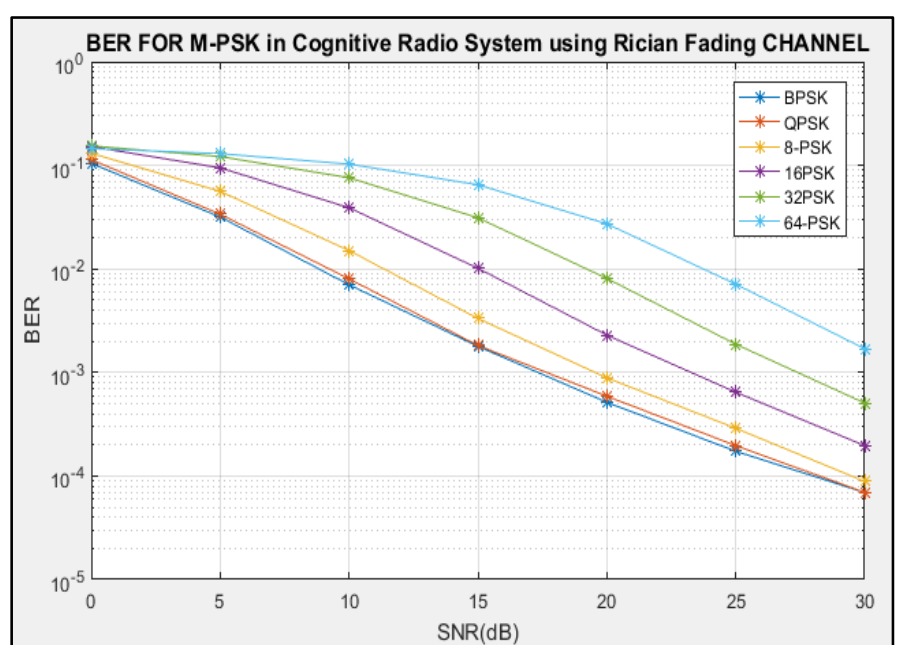

Figure (6): BER FOR ALL M-PSK\&QPSK in Rician fading channel.

Figure (6) shows performance analysis of M-PSK modulation technique over Rician fading channel AWGN and. BPSK\&QPSK Has the lowest rate of errors (BER) compared to other types of M-PSK. At SNR of $20 \mathrm{~dB}$ the average BER in BPSK\& QPSK is less than $10^{-3}(0.001)$ and the BER in other M-PSK types: 8,16 , $32 \& 64-P S K$ is greater than $10^{-3}(0.001)$. Finally the BER performance of low level M-PSK is better than high level of M-PSK, and when data rate increase the BER increase this is clear in case of 64-PSK compared with the BPSK \& QPSK.

\section{Conclusion}

The performance of CR system based on energy detection to detect the holes in a licensed band of PU to be used by SU over AWGN and Rician fading channels have been done. The analysis is based on study of BER VS SNR. From the results obtained as BER decreases as the SNR increases. The low level of M-PSK better performance as compared to high level M-ary PSK techniques. If spectral efficiency is not considered or taken into consecration of the performance. 


\section{References}

[1]. Julian Rubin (2013) The Discovery of Electromagnetic at: http://www.juliantrubin.com/bigten/hertzexperiment.html (Accessed: 5-5-2017).

[2]. J. Mitola III and G. Q. Maguire, Jr., "Cognitive radio: Making software radios more personal," IEEE Personal Communications Magazine, vol. 6, no. 4, pp. 13-18, Aug. 1999. DOI: 10.1109/98.788210.

[3]. I. F. Akyildiz, L. Won-Yeol, and K.R. Chowdhury. Spectrum management in cognitive radio ad hoc networks. IEEE Xplore, 3(12):6 12, 2009.

[4]. R.Tandra, S. M. Mishra, and A. Sahai. What is a spectrum hole and what does it take to recognize one? Proceedings of the IEEE, 97(5):824-848, 2009].

[5]. Ghayoor Abbas Jafri ,Ateeq Ur Rehman ,Muhammad Tariq Sadiq "Spectrum Sensing and Management in Cooperative Cognitive Radio" thesis presented as part of Master Degree of Science in Electrical Engineering Blekinge Institute of Technology ,May 2011.

[6]. Andrea Goldsmith, "Wireless Communications", Cambridge University Press, 2005.

[7]. Behrouz Foroujan, "Data Communications \& Networking", 4th Edition, the McGraw-Hill Companies.

[8]. T.S Rappaport. "Wireless Communication Principle \&Practice", $2^{\text {nd }}$ Edition, Prentice Hall, 2003.

[9]. Frenzel, Louis E. "Principles of Electronics Communication Systems", 3rd edition, McGraw Hill, 2008.

[10]. Young, Paul H "Electronic Communications Techniques" 5th edition, Prentice Hall, 2004

[11]. JOHN B. ANDERSON "Digital Transmission Engineering” Second Edition, Lund University.

[12]. Fuqin Xiong "Digital modulation techniques", 2006.

[13]. Lindsey, W.C.: Error probabilities for Rician fading multichannel reception of binary and n-ary Signals. IEEE Trans. Inf. Theory IT-10(4), 333-350 (1964) 